\title{
In vitro Thrombolytic and Membrane Stabilizing Studies of Brassica rapa subsp. chinensis (L.) Hanelt
}

\author{
Nushrat Jahan ${ }^{1}$, S. M. Ashikur Rahman ${ }^{1}$, Mohammad S. Rahman ${ }^{2}$ and \\ Mohammad A. Rashid ${ }^{2}$ \\ ${ }^{1}$ Department of Pharmacy, State University of Bangladesh, Dhaka-1205, Bangladesh \\ ${ }^{2}$ Phytochemical Research Laboratory, Department of Pharmaceutical Chemistry, Faculty of Pharmacy, \\ University of Dhaka, Dhaka-1000, Bangladesh
}

Received: June 23, 2014;

Accepted: July 21, 2014;

Published (Web): July 23, 2014

\begin{abstract}
Brassica rapa subsp. chinensis (L.) Hanelt is a popular herb in Bangladesh. In the present studies, the plant has been assessed to evaluate its in vitro thrombolytic and membrane stabilizing properties. Initially, the plant was collected, processed and extracted with methanol and the concentrated crude methanol extract was fractionated into petroleum ether, carbon tetrachloride, chloroform and aqueous soluble materials. All extractives, including the parent extract were subjected to thrombolytic and membrane stabilizing bioassays. In case of thrombolytic study, the carbon tetrachloride soluble fraction exhibited highest clot lysis. Here, streptokinase and distilled water were used as positive and negative control, respectively. However, in the assay for membrane stabilizing activity, the crude methanol extract along with other extractives were capable to inhibit hemolysis of erythrocyte membrane in hypotonic solution- and heat- induced conditions, which indicated anti-inflammatory property of the samples. Acetyl salicylic acid was used as standard drug in this assay. This represents the first report of thrombolytic and membrane stabilizing activities of $B$. rapa subsp. chinensis.
\end{abstract}

Key words: Brassica rapa subsp. chinensis, Brassicaceae, membrane stabilization, thrombolytic, antiinflammatory

\section{Introduction}

Medicinal plants have been proven to be very promising candidates for developing new drugs. Plants have wide range of potentials for treating different types of diseases. Systematic screening of plants might be a good tool for finding and isolating bioactive lead molecules (El-Abhar and Schaalan, 2014; Jin et al., 2014; Naveen et al., 2014).

Thrombosis and inflammation are frequently observed in human body for making very complicated situations. Many drugs have been developed to take care of these problems but side effects still remain as a matter of concern (Fox and Kahn, 2005; Rodriguez et al., 2012; Wilson and Chaikof, 2008). To obtain safer molecules, comprehensive studies are still going on. Natural products might be very useful resources for finding the desired drug.

Bangladesh is in the tropical zone blessed with thousands of plants and people here are accustomed with herbal practices for long time (Chowdhury et al., 2010;
Jahan et al., 2010; Rahman et al., 2011). Plants growing here have not been systemically evaluated yet to a significant extent. With this view, B. rapa subsp. chinensis was selected here to explore its medicinal properties.

B. rapa subsp. chinensis (L.) Hanelt (Bengali name: Bati shak) belongs to the Brassicaceae family. The leaves are commonly light green and thin. White-fleshed roots are developed at the base of the leaf petioles. At the top of the raceme the flowers remain as cluster. This plant is usually famous as vegetable, which is very low in calories. It is a very good source of many nutrients, vitamins, minerals and antioxidants (Dominguez-Perles et al., 2014; Siddiqui et al., 2014). Previous phytochemical investigations on Brassica genus led to the isolation of various types of phenolics and organic acids (Fernandes et al., 2007; Mucha-Pelzer et al., 2010; Romani et al., 2006; Tenore et al., 2012).

The aim of the current study was to evaluate the $B$. rapa subsp. chinensis for thrombolytic and membrane stabilizing activities for the first time. 


\section{Materials and Methods}

Collection of plant materials: The whole plants of Brassica rapa subsp. chinensis were collected from Gazipur, Bangladesh on June 2013 and a voucher specimen (DACB accession no. 39457) has been deposited at Bangladesh National Herbarium, Mirpur, Dhaka for future reference.

Extraction: The collected plant materials were chopped, dried and powdered. About $500 \mathrm{~g}$ of the powdered materials was soaked in 1.5 litre of methanol at room temperature for 7 days. The extract was filtered through cotton plug and concentrated with a rotary evaporator. An aliquot (5 g) of the concentrated methanol extract was fractionated by the modified Kupchan method (VanWagenen et al., 1993) into petroleum ether, carbon tetrachloride, chloroform and aqueous soluble fractions. Subsequent evaporation of solvents yielded petroleum ether (PE, $0.80 \mathrm{~g})$, carbon tetrachloride (CT, $1.80 \mathrm{~g}$ ), chloroform (CF $1.20 \mathrm{~g}$ ) and aqueous (AQ $1.20 \mathrm{~g}$ ) soluble materials, respectively.

Thrombolytic activity: In case of in vitro thrombolytic assay (Prasad et al., 2006; Prasad et al., 2007), $5 \mathrm{ml}$ of venous blood was drawn from healthy volunteers, distributed in different pre-weighed sterile microcentrifuge tubes $(0.5 \mathrm{ml} /$ tube $)$ and then incubated at $37{ }^{\circ} \mathrm{C}$ for 45 minutes. After clot formation, the serum was completely removed without disturbing the clot and each tube having clot was again weighed to determine the weight of the clot. To each tube, each of the extractives $(1 \mathrm{mg} / 100 \mu \mathrm{l}$ water) was added. Here, $100 \mu$ (equivalent to 30,000 I.U.) of streptokinase (Altepase ${ }^{\circledR}$, Beacon Pharmaceuticals Limited, Bangladesh) and $100 \mu \mathrm{l}$ of distilled water were used as positive control and negative control, respectively. After incubation of the tubes at $37^{\circ} \mathrm{C}$ for 90 minutes, the release of fluid from the clot was removed and tubes were again weighed to observe the difference in weight after clot disruption. Percentage of clot lysis was expressed as: $\%$ thrombolysis $=($ weight of clot after treatment $/$ weight of clot before treatment $) \times 100$

Membrane stabilizing activity: The following two methods were used for conducting this in vitro membrane stabilizing assay (Shinde et al., 1999; Sikder et al., 2012).

i) Hypotonic solution-induced hemolysis: To prepare the erythrocyte suspension, $5 \mathrm{ml}$ of whole blood was obtained from healthy human volunteers in a tube containing dipotassium salt of EDTA $(2.2 \mathrm{mg} / \mathrm{ml}$ of blood). The blood was centrifuged, supernatant was removed and blood cells were washed three times by sodium chloride isotonic solution $(154 \mathrm{mM} \mathrm{NaCl})$ in 10 $\mathrm{mM}$ sodium phosphate buffer $(\mathrm{pH}$ 7.4) through centrifugation (10 min at $3000 \mathrm{~g}$ ) using the same volume as supernatant. Finally, it was resuspended in the same volume of this isotonic buffer solution. After that, $0.5 \mathrm{ml}$ of this was mixed with $5 \mathrm{ml}$ of hypotonic solution $(50 \mathrm{mM}$ $\mathrm{NaCl})$ in $10 \mathrm{mM}$ sodium phosphate buffered saline $(\mathrm{pH}$ 7.4) containing either the extract $(2 \mathrm{mg} / \mathrm{ml})$ or reference drug, acetyl salicylic acid $(0.1 \mathrm{mg} / \mathrm{ml})$. The control sample was consisted of $0.5 \mathrm{~mL}$ of RBCs mixed with hypotonicbuffered saline alone. The mixture was incubated for 10 $\mathrm{min}$ at room temperature, centrifuged for $10 \mathrm{~min}$ at $3000 \mathrm{~g}$ and the optical density (OD) of the supernatant was measured at $540 \mathrm{~nm}$. The percentage inhibition of hemolysis was calculated using the following equation-

$$
\begin{gathered}
\% \text { inhibition of hemolysis }=\left\{\left(\mathrm{OD}_{\text {control }}-\mathrm{OD}_{\text {test sample }}\right) /\right. \\
\left.\mathrm{OD}_{\text {control }}\right\} \times 100
\end{gathered}
$$

ii) Heat-induced hemolysis: $5 \mathrm{ml}$ of isotonic buffer containing aliquots $(1 \mathrm{mg} / \mathrm{ml})$ of the different extractives were put into two duplicate sets of centrifuge tubes. The vehicle $(5 \mathrm{ml})$ was used as control in a separate tube. Afterwards, $30 \mu \mathrm{l}$ erythrocyte suspension (prepared similarly as in hypotonic solution induced hemolysis method) was added to each tube and mixed gently. One pair of the tubes and control samples were incubated at 54 ${ }^{\circ} \mathrm{C}$ for $20 \mathrm{~min}$ in a water bath, while the other pair was maintained at $0-5{ }^{\circ} \mathrm{C}$ in an ice bath. The reaction mixture was centrifuged for $3 \mathrm{~min}$ at $1300 \mathrm{~g}$ and the absorbance of the supernatant was measured at $540 \mathrm{~nm}$. The percentage inhibition or acceleration of hemolysis in tests was calculated according to the equation:

$$
\begin{aligned}
& \text { \% Inhibition of hemolysis }=\left\{1-\left(\mathrm{OD}_{\text {heated test sample }}-\right.\right. \\
& \left.\quad \mathrm{OD}_{\text {unheated test sample }}\right) / \\
& \left.\left(\mathrm{OD}_{\text {heated control sample }}-\mathrm{OD}_{\text {heated test sample }}\right)\right\} \times 100
\end{aligned}
$$

Statistical analysis: Three replicates $(\mathrm{n}=3)$ of each sample were used for statistical analysis and the values are reported as mean \pm standard deviation (SD).

\section{Results and Discussion}

Thrombolytic activity: The thrombolytic activity of the plant extractives are shown in Figure 1. The highest thrombolytic activity (58.57\%) was noticed by the carbon 
tetrachloride soluble fraction of the crude methanolic extract. However, moderate clot lysis ability (considering $>40 \%$ lysis) was also exhibited by the chloroform (49.39 $\%)$ and petroleum ether (43.37\%) soluble fractions. However, the crude methanolic extract and its aqueous soluble fraction showed weaker thrombolytic activity.

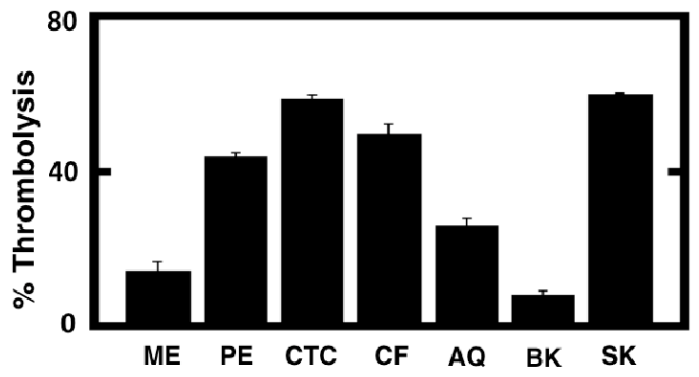

Figure 1: Effect of different extractives of $B$. rapa subsp. chinensis on thrombolysis. Here, ME: crude methanolic extract; PE- petroleum ether soluble fraction of methanolic extract; CTC- carbon tetrachloride soluble fraction of methanolic extract; CF- chloroform soluble fraction of methanolic extract; AQ-aqueous fraction; BK-blank (negative control); SK-streptokinase (positive control).

Membrane stabilizing activity: The membrane stabilizing activities are depicted in Figure 2. In case of hypotonic solution induced hemolysis (Figure 2A), the maximum level of membrane stabilizing activity was observed by the crude methanolic extract (78.31\%). Besides, all the partitionates of the crude methanolic extract except petroleum ether soluble fraction displayed more than $70 \%$ of the membrane protecting capabilities.

In case of heat induced hemolysis (Figure 2B), the crude methanol extract and its chloroform soluble fraction exhibited stronger protection to the membrane (>80\% inhibition of hemolysis). Among all other partitionates, the carbon tetrachloride and aqueous soluble extractives showed more than $55 \%$ inhibition of the heat induced hemolysis of membrane.

Bangladesh is a country which is blessed with numerous medicinal plants. For thousands of years, people have been using plants for traditional healing. Many of them are considered beneficial but there is a great lack of systematic evaluation to prove the efficacy of the plant remedies. In this consideration, scientific evaluation is the demand of time to explore the medicinal functional aspects of these plants (Islam et al., 2009; Kabir et al., 2010; Rahman et al., 2008a; Rahman et al., 2008b). Brassica is a genus encompassing lot of species. People are used to take this plant as vegetables in Bangladesh and many other countries. Nutritionally these herbs are considered very much exclusive (Dominguez-Perles et al., 2014; Fernandes et al., 2007). Therefore, it is a good opportunity to study this plant for evaluating other health benefits. In the current studies, among many Brassica speices, B. rapa subsp. chinensis (L.) was subjected to the thrombolytic and membrane stabilizing assays for the first time.
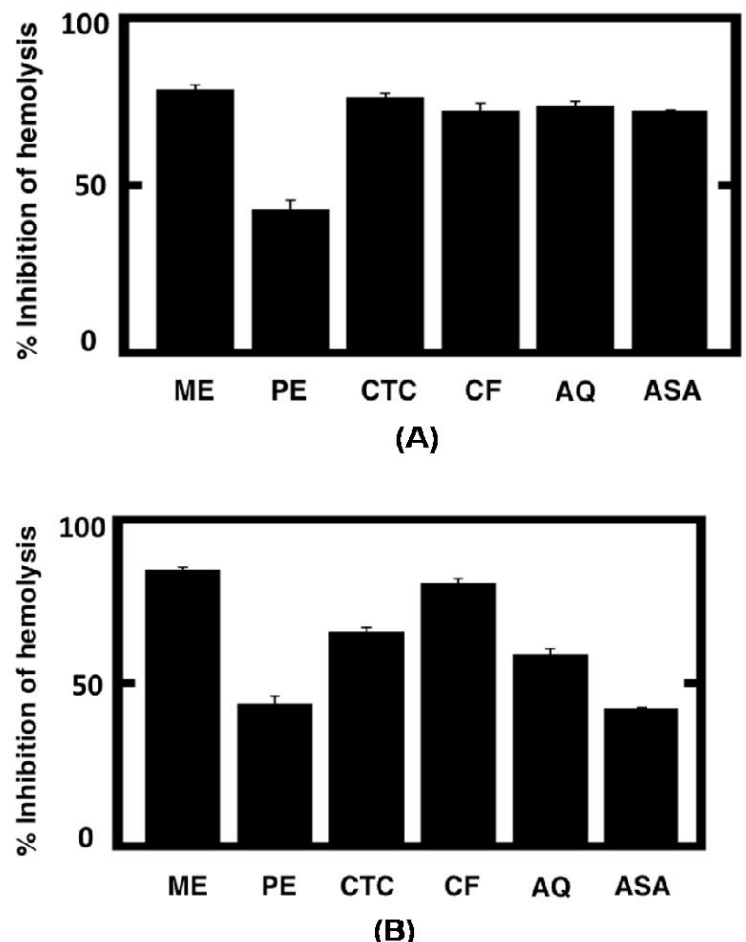

Figure 2: Effect of different extractives of $B$. rapa subsp. chinensis on hypotonic solution induced (A) and heat induced (B) hemolysis of erythrocyte membrane. In both cases, ME: crude methanolic extract; PE- petroleum ether soluble fraction of methanolic extract; CTC- carbon tetrachloride soluble fraction of methanolic extract; $\mathrm{CF}$ - chloroform soluble fraction of methanolic extract; AQ- aqueous fraction; ASA- acetyl salicylic acid (standard drug).

Thrombus formation in the circulatory system causes vascular blockage leading to death. Currently used thrombolytic agents that include tissue plasminogen activator, urokinase, streptokinase etc. are used all over the world but still associated with risk of hemorrhage, anaphylactic reaction and lack specificity. So, attempts are still ongoing around the world to develop improved thrombolytic agents (Hilleman and Campbell, 2011; Tsikouris and Tsikouris, 2001; van Domburg et al., 2000; 
Woo and White, 1993). With this view, this investigation was done on B. rapa subsp. chinensis. The carbon tetrachloride, chloroform and petroleum ether soluble partitionates of the crude methanol extract showed potent thrombolytic activity (Figure 1). It was previously reported that polyphenols and flavonoids have significant thrombolytic activity (Chen et al., 2012; Fuentes et al., 2014). B. rapa subsp. chinensis is also rich in polyphenols and flavonoids as secondary metabolites (Fernandes $\mathrm{et}$ al., 2007; Romani et al., 2006). This might be a reason to display noticeable thrombolytic activity of this plant.

Inflammation is the pathology to make people suffer. Numerous anti-inflammatory drugs are available in the market but all remain with some side effects more or less. If dietary agents can provide some anti-inflammatory effects, it might be very beneficial in the time of need. Membrane-stabilizing experiment can serve as an indicator to screen out the anti-inflammatory agents. Compounds with membrane-stabilizing properties can prevent the release of phospholipases that initiate the formation of inflammatory mediators (Shinde et al., 1999). In this regard, the experimental herb was subjected to membrane stabilizing assay in order to check its performance to prevent hemolysis by both hypotonic solution- and heat- induction. The extractives of $B$. rapa subsp. chinensis (L.) demonstrated significant membrane stabilizing property (Figure 2). It is noteworthy to mention that this plant possesses a lot of phenolics and flavonoids (Fernandes et al., 2007; Romani et al., 2006; Tenore et al., 2012), which may be considered as causative agents to show substantial anti-inflammatory activity (Jean-Gilles et al., 2012; Jin et al., 2010; Pan et al., 2010; Santangelo et al., 2007). Even this plant is taken as vegetable, medicinal properties may add some values over nutritional contribution.

\section{Conclusion}

B. rapa subsp. chinensis (L.) extractives were evaluated for the first time to check their thrombolytic and membrane stabilization potential. The plant demonstrated potent thrombolytic and membrane stabilization activities. Even though, this herb is generally considered as vegetable, the current studies have proven it to be a useful remedial plant. Further comprehensive investigations are required to isolate the bioactive compounds and to know their detailed underlying mechanism.

\section{References}

Chen, C., Li, S.X., Wang, S.M. and Liang, S.W. 2012. Investigation into the anti-thrombosis effect and contents of total saponins and flavonoids in the bioactive fraction of Naodesheng prescription. J. Ethnopharmacol. 144, 208212.

Chowdhury, A., Alam, M.A., Rahman, M.S., Hassan, M.A. and Rashid, M.A. 2010. Antioxidant, Antimicrobial and Cytotoxic Activities of Corypha taliera Roxb. Latin Am. J. Pharm. 29, 1231-1234.

Dominguez-Perles, R., Mena, P., Garcia-Viguera, C. and Moreno, D.A. 2014. Brassica foods as a dietary source of vitamin C: a review. Crit. Rev. Food Sci. Nutr. 54, 10761091.

El-Abhar, H.S. and Schaalan, M.F. 2014. Phytotherapy in diabetes: Review on potential mechanistic perspectives. World J. Diabetes 5, 176-197.

Fernandes, F., Valentão, P., Sousa, C., Pereira, J.A., Seabra, R.M. and Andrade, P.B. 2007. Chemical and antioxidative assessment of dietary turnip (Brassica rapa var. rapa L.). Food Chem. 105, 1003-1010.

Fox, E.A. and Kahn, S.R. 2005. The relationship between inflammation and venous thrombosis. A systematic review of clinical studies. Thromb. Haemost. 94, 362-365.

Fuentes, E., Guzmán, L., Alarcón, M., Moore, R. and Palomo, I., 2014. Thrombolytic/Fibrinolytic mechanism of natural products. Fibrinolysis and thrombolysis, Dr. Krasimir Kolev (Ed.), ISBN: 978-953-51-1265-5, InTech, DOI 10.5772/57608. Available from: http://www.intechopen com/books/fibrinolysis-and-thrombolysis/thrombolyticfibrinolytic-mechanism-of-natural-products

Hilleman, D. and Campbell, J. 2011. Efficacy, safety, and cost of thrombolytic agents for the management of dysfunctional hemodialysis catheters: a systematic review. Pharmacotherapy 31, 1031-1040.

Islam, M.R., Uddin, M.Z., Rahman, M.S., Tutul, E., Rahman, M.Z., Hassan, M.A., Faiz, M.A., Hossain, M., Hussain, M. and Rashid, M.A. 2009. Ethnobotanical, phytochemical and toxicological studies of Xanthium strumarium L. Bangladesh Med. Res. Counc. Bull. 35, 84-90.

Jahan, I., Rahman, M.S., Rahman, M.Z., Kaisar, M.A., Islam, M.S., Wahab, A. and Rashid, M.A. 2010. Chemical and biological investigations of Delonix regia (Bojer ex Hook.) Raf. Acta Pharm. 60, 207-215.

Jean-Gilles, D., Li, L., Ma, H., Yuan, T., Chichester, C.O., 3rd and Seeram, N.P. 2012. Anti-inflammatory effects of polyphenolic-enriched red raspberry extract in an antigeninduced arthritis rat model. J. Agric. Food Chem. 60, 57555762 . 
Jin, G.L., Su, Y.P., Liu, M., Xu, Y., Yang, J., Liao, K.J. and Yu, C.X. 2014. Medicinal plants of the genus Gelsemium (Gelsemiaceae, Gentianales)- a review of their phytochemistry, pharmacology, toxicology and traditional use. J. Ethnopharmacol. 152, 33-52.

Jin, J.H., Kim, J.S., Kang, S.S., Son, K.H., Chang, H.W. and Kim, H.P. 2010. Anti-inflammatory and anti-arthritic activity of total flavonoids of the roots of Sophora flavescens. J. Ethnopharmacol. 127, 589-595.

Kabir, S., Rahman, M.S., Chowdhury, A.M., Hasan, C.M. and Rashid, M.A. 2010. An unusual bisnor-clerodane diterpenoid from Polyalthia simiarum. Nat. Prod. Commun. 5, 1543-1546.

Mucha-Pelzer, T., Mewis, I. and Ulrichs, C. 2010. Response of Glucosinolate and Flavonoid Contents and Composition of Brassica rapa ssp. chinensis (L.) Hanelt to Silica Formulations Used as Insecticides. J. Agric. Food Chem. 58, 12473-12480.

Naveen, Y.P., Divya Rupini, G., Ahmed, F. and Urooj, A. 2014. Pharmacological effects and active phytoconstituents of Swietenia mahagoni: a review. J. Integr. Med. 12, 86-93.

Pan, M.H., Lai, C.S. and Ho, C.T. 2010. Anti-inflammatory activity of natural dietary flavonoids. Food Funct. 1, 15-31.

Prasad, S., Kashyap, R.S., Deopujari, J.Y., Purohit, H.J., Taori, G.M. and Daginawala, H.F. 2006. Development of an in vitro model to study clot lysis activity of thrombolytic drugs. Thromb. J. 4, 14.

Prasad, S., Kashyap, R.S., Deopujari, J.Y., Purohit, H.J., Taori, G.M. and Daginawala, H.F. 2007. Effect of Fagonia arabica (Dhamasa) on in vitro thrombolysis. BMC Complement. Altern. Med. 7, 36.

Rahman, M.S., Begum, B., Chowdhury, R., Rahman, K.M. and Rashid, M.A. 2008a. Preliminary cytotoxicity screening of some medicinal plants of Bangladesh. Dhaka Univ. J. Pharm. Sci. 7, 47-52.

Rahman, M.S., Rahman, M.Z., Wahab, M.A., Chowdhury, R. and Rashid, M.A. 2008b. Antimicrobial activity of some indigenous plants of Bangladesh. Dhaka Univ. J. Pharm. Sci., 7, 23-26.

Rahman, M.S., Rahman, M.Z., Begum, B., Chowdhury, R., Islam, S.N. and Rashid, M.A. 2011. Antidiabetic principle from Eclipta prostrata. Latin Am. J. Pharm. 30, 1656-1660.

Rodriguez, A.L., Wojcik, B.M., Wrobleski, S.K., Myers, D.D., Jr., Wakefield, T.W. and Diaz, J.A. 2012. Statins, inflammation and deep vein thrombosis: a systematic review. J. Thromb. Thrombolysis 33, 371-382.
Romani, A., Vignolini, P., Isolani, L., Ieri, F. and Heimler, D. 2006. HPLC-DAD/MS characterization of flavonoids and hydroxycinnamic derivatives in turnip tops (Brassica rapa L. Subsp. sylvestris L.). J. Agric. Food Chem. 54, 13421346.

Santangelo, C., Vari, R., Scazzocchio, B., Di Benedetto, R., Filesi, C. and Masella, R. 2007. Polyphenols, intracellular signalling and inflammation. Ann. Ist Super Sanita 43, $394-$ 405.

Shinde, U.A., Phadke, A.S., Nair, A.M., Mungantiwar, A.A., Dikshit, V.J. and Saraf, M.N. 1999. Membrane stabilizing activity - a possible mechanism of action for the antiinflammatory activity of Cedrus deodara wood oil. Fitoterapia 70, 251-257.

Siddiqui, M.M., Abbasi, B.H., Ahmad, N., Ali, M. and Mahmood, T. 2014. Toxic effects of heavy metals (Cd, Cr and $\mathrm{Pb}$ ) on seed germination and growth and $\mathrm{DPPH}-$ scavenging activity in Brassica rapa var. turnip. Toxicol. Ind. Health 30, 238-249.

Sikder, M.A.A., Kaisar, M.A., Rashid, M.A., Millat, M.S. and Sultana, A. 2012. In vitro membrane stabilizing activity, total phenolic content, cytotoxic, thrombolytic and antimicrobial activities of Calliandra surinamensis (Wall.). J. Pharmacog. Phytochem. 1, 45-50.

Tenore, G.C., Troisi, J., Di Fiore, R., Basile, A. and Novellino, E. 2012. Chemical composition, antioxidant and antimicrobial properties of Rapa Catozza Napoletana (Brassica rapa L. var. rapa DC.) seed meal, a promising protein source of Campania region (southern Italy) horticultural germplasm. J. Sci. Food Agric. 92, 1716-1724.

Tsikouris, J.P. and Tsikouris, A.P. 2001. A review of available fibrin-specific thrombolytic agents used in acute myocardial infarction. Pharmacotherapy 21, 207-217.

van Domburg, R.T., Boersma, E. and Simoons, M.L. 2000. A review of the long term effects of thrombolytic agents. Drugs 60, 293-305.

VanWagenen, B.C., Larsen, R., Cardellina, J.H., Randazzo, D., Lidert, Z.C. and Swithenbank, C. 1993. Ulosantoin, a potent insecticide from the sponge Ulosa ruetzleri. J. Org. Chem. 58, 335-337.

Wilson, J.T. and Chaikof, E.L. 2008. Thrombosis and inflammation in intraportal islet transplantation: a review of pathophysiology and emerging therapeutics. J. Diabetes Sci. Technol. 2, 746-759.

Woo, K.S. and White, H.D. 1993. Comparative tolerability profiles of thrombolytic agents. A review. Drug Saf. 8, 19-29. 